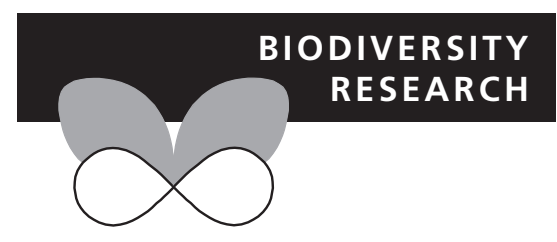

\title{
The importance of parasite geography and spillover effects for global patterns of host-parasite associations in two invasive species
}

\author{
Konstans Wells ${ }^{1 \star}$, Robert B. O’Hara ${ }^{2}$, Serge Morand ${ }^{3}$, \\ Jean-Philippe Lessard ${ }^{4,5}$ and Alexis Ribas ${ }^{6}$
}

${ }^{1}$ The Environment Institute, School of Earth and Environmental Sciences, The University of Adelaide, Adelaide, SA, Australia, ${ }^{2}$ Biodiversity and Climate Research Centre (BiK-F), Frankfurt, Germany, ${ }^{3}$ Centre d'Infectiologie Christophe Mérieux du Laos, CIRAD AGIRs, CNRS ISEM, Vientiane, Lao People's Democratic Republic, ${ }^{4} Q u e ́ b e c$ Centre for Biodiversity Science, McGill University, Montréal, QC, Canada,

${ }^{5}$ Department of Biology, Concordia University, Montréal, QC, Canada, ${ }^{6}$ Biodiversity Research Group, Faculty of Science, Udon Thani Rajabhat University, Udon Thani, Thailand

${ }^{*}$ Correspondence: Konstans Wells, School of Earth \& Environmental Sciences and the Environment Institute, The University of Adelaide, North Terrace, Adelaide SA 5005, Australia.

E-mail: konstans.wells@adelaide.edu.au

\begin{abstract}
Aim Geographic spread and range expansion of species into novel environments may merge originally separated species assemblages, yet the possible drivers of geographic heterogeneity in host-parasite associations remain poorly understood. Here, we examine global patterns in the parasite assemblages of two rat species and explore the role of parasite acquisition from local pools of host species.
\end{abstract}

Location Global.

Methods We compiled a global data set of helminth parasites ( $n=241$ species) from two rat species (Rattus rattus species complex, R. norvegicus) and, concomitantly, from all other mammal species known to be infected by the same parasites. We used an inverse Bayesian modelling approach to explicitly link species-level to community-level infestation probabilities at different geographic scales and alleviate the shortcoming of sampling bias.

Results Patterns of species richness and turnover of parasites in the two focal rat species revealed clear biogeographic structure with lowest species richness and most distinct assemblages in Madagascar and highest species richness and least distinct assemblages in the Palaearctic region. Parasite species richness and turnover across regions were correlated for the two focal hosts, although they were associated with distinct assemblages within regions. Infection probability of a focal host with any given parasite was clearly related to infection probability of the local species pool of wildlife hosts with that same parasite. Infection probability of other mammal species infected with these parasite species, in turn, decreased with their taxonomic distance to the genus Rattus.

Main conclusions Our study demonstrates the importance of spillover of parasites from local wildlife hosts to invasive rats on global patterns of host-parasite associations. Considering both changes in local pools of host species and the global distributions of parasite and pathogen diversity in consistent model frameworks may therefore advance the forecasting of species-level infestation patterns and the possible risk of disease emergence from local to global scale.

\section{Keywords}

Biogeographic regions, biological invasions, geographic mosaics, global diversity, helminths, host-parasite associations, inverse modelling, parasite spread, species distribution, zoonoses. 


\section{INTRODUCTION}

As much as $60 \%$ of human diseases are of zoonotic origin (Taylor et al., 2001), but our knowledge of how parasites are distributed and shared among wildlife, commensal and domestic animal species is inevitably incomplete given the challenge to exhaustively document possible host-parasite combinations for thousands of species. Moreover, while it is evident that environmental change alters conditions for parasite persistence and transmission (Patz et al., 2000), we lack a solid understanding of how global patterns in host-parasite associations are shaped by geographic range limits of parasites and interactions between invasive hosts and native assemblages of wildlife hosts (Morand \& Krasnov, 2010; Estrada-Peña et al., 2014).

During historical dispersal and invasions of new environments, host species are likely to escape from some associated parasite species and thus harbour fewer parasites in newly colonized regions compared to the associated parasite assemblages in their native range (Poulin \& Mouillot, 2003; Torchin et al., 2003). Moreover, a local assemblage of parasites (i.e. all parasites found in a host species in a region) infecting a widely distributed host species (e.g. commensal rat) may be strongly influenced by acquisition from the local pool of wildlife hosts, that is a gain of parasites that originated in local wildlife species (Daszak et al., 2000). Geographical structure in host-parasite associations is thus likely to track patterns of wildlife diversity such as those observed along broad-scale environmental gradients (Jenkins et al., 2013) and on global maps of zoogeographic regions (Holt et al., 2013). The total species richness of parasites in local host communities often correlates positively with the species richness of hosts (Krasnov et al., 2004; Thieltges et al., 2011). As such, an invasive host species colonizing an area with a high diversity of wildlife species is likely to be exposed to a high diversity of potentially suitable parasite species. However, increasing the diversity of host species may also cause unfavourable conditions for parasites if host species differ in quality. In such cases, increasing host species richness can reduce parasite transmissibility due to more encounters with unfavourable hosts (Ostfeld \& Keesing, 2012). The strength and generality of the relationship between the number of parasites in an invasive host species and the diversity of local wildlife assemblages as potential reservoirs over large geographic scales remain therefore elusive (Morand, 2012).

Uncertainty persists as to whether parasite diversity on any given species of host in a local community is positively related to local host diversity. Presumably, the parasite species richness of any given host species should be highest in its ancestral centre of origin (i.e. South and Southeast Asia for commensal rats of the genus Rattus; Robins et al., 2008; Aplin et al., 2011). The sharing of parasite species with other species from local host species pools can be expected to be highest if species have a long history of sharing the same biogeographical space: the longer domestic and commensal animals are associated with humans, for example the more parasites they share with them (Morand et al., 2014).

In this study, we explored changes in parasite species richness and turnover at global scale and the role of parasite acquisition from local pools of wildlife hosts of two of the most cosmopolitan invaders and important commensal rat species. The black rat Rattus rattus (species complex) and the Norway rat Rattus norvegicus have been introduced in most regions of the world as a result of human activities (Aplin et al., 2011), have a long history of disease transmission to humans (Meerburg et al., 2009) and cause considerable economic loss (Singleton et al., 2003; Stenseth et al., 2003). $R$. rattus invades a large range of semi-natural and natural environments, where it is likely to interact with various wildlife species (Goodman, 1995; Harris et al., 2006; Wells et al., 2014). Such human-induced mixture of anthropogenic and natural habitats and animal species are likely to enhance the exchange of parasite species across environments (Hoberg, 2010). R. norvegicus is more strongly associated with urban environments that generally harbour fewer wildlife species (Wells et al., 2014). We may therefore expect parasite assemblages of $R$. rattus to reflect the higher richness of reservoir hosts in their environment relative to that of $R$. norvegicus. The two rat species could be expected to share similar parasite assemblages and exhibit similar patterns of spatial turnover across zoogeographic regions if we take into account that they occur in sympatry in urban environments and parasite may frequently shift between these two closely related species.

Not only do we know very little about global geographic trends of host-parasite associations; there are important methodological obstacles that can preclude obtaining a clear picture. Species distributional data commonly include bias towards heterogeneous sampling efforts and incomplete sampling (Lomolino, 2004; Hortal et al., 2007; Boakes et al., 2010). Incomplete inventories introduce 'false' zeros into data (Martin et al., 2005), and there is uncertainty as to whether host-parasite associations are lacking or have simply been unobserved (Hopkins \& Nunn, 2007). Especially in comparative studies, sampling bias and incomplete inventories may lead to misleading conclusions about host-parasite associations if not accurately accounted for in analyses (Wells et al., 2013). We must therefore develop analytical tools that will minimize how sampling biases influence our perception of geographic patterns in host-parasite associations.

Addressing our study question with incomplete observations inevitably calls for statistical approaches that take uncertainty and unknown measures into account (Keating \& Cherry, 2004; Reese et al., 2005; Ward et al., 2009). We fitted an inverse modelling approach in a Bayesian hierarchical framework to estimate possible host-parasite associations from a limited set of observations, while also accounting for the possible links between parasite species and local species pools of wildlife hosts.

We therefore used the flexibility of a hierarchical Bayesian approach for estimating parasite occurrence at poorly sampled 
locations by 'borrowing strength' from more intensively sampled locations, while also acknowledging that locations are not identical in all aspects. The hierarchical model structure further allows to model the variation of parasite occurrence in wildlife hosts according to species and population attributes and environmental variables (Fig. 1). For example, we can ask whether species of conservation concern are particularly sensitive to share parasites with invasive (focal) species, fostering our understanding for informed wildlife management and pest control (Daszak et al., 2000). We systematically combined information at the species level (i.e. parasite associations in the focal rats species) with those at the community level (i.e. wildlife hosts linked to rats by sharing the same parasites) into a hierarchical model that optimizes inference by maximizing the use of all available information and simultaneously assessing the influence of ecological processes expected to operate across levels of organization.

\section{METHODS}

\section{Database on host-parasite records}

We compiled a database of recorded associations between the focal rat species and their helminth parasites from the host-parasite database of the Natural History Museum London (NHML) (Gibson et al., 2005), which includes host-parasite records from more than 28,000 references up to 2003 (accessed in June 2013).

For each field record (excluding experimental and captive records), we characterized the geographic location based on current country-level geographic borders. We specified this characterization in subregions for some locations such as China (which encompasses multiple zoogeographic regions; for all records from China which could not be identified to subregion, we used an extra category that specified zoogeographic region as missing data). Additionally we separated records from different islands in Indonesia (e.g. we considered Borneo as a separate location irrespective of whether records were made in the Indonesian or Malaysian part of the island). For countries with few records, we merged neighbouring countries into larger units such as Scandinavia (Finland, Norway, Sweden). We are aware that this classification is coarse and arbitrary. Nevertheless, we consider this approach to be acceptable in order to systematically assign all records to geographical units while accounting for the global topography and zoogeographic structure of a large set of records with no detailed geographic positions available. Our data set for analysis included 144 geographic locations.

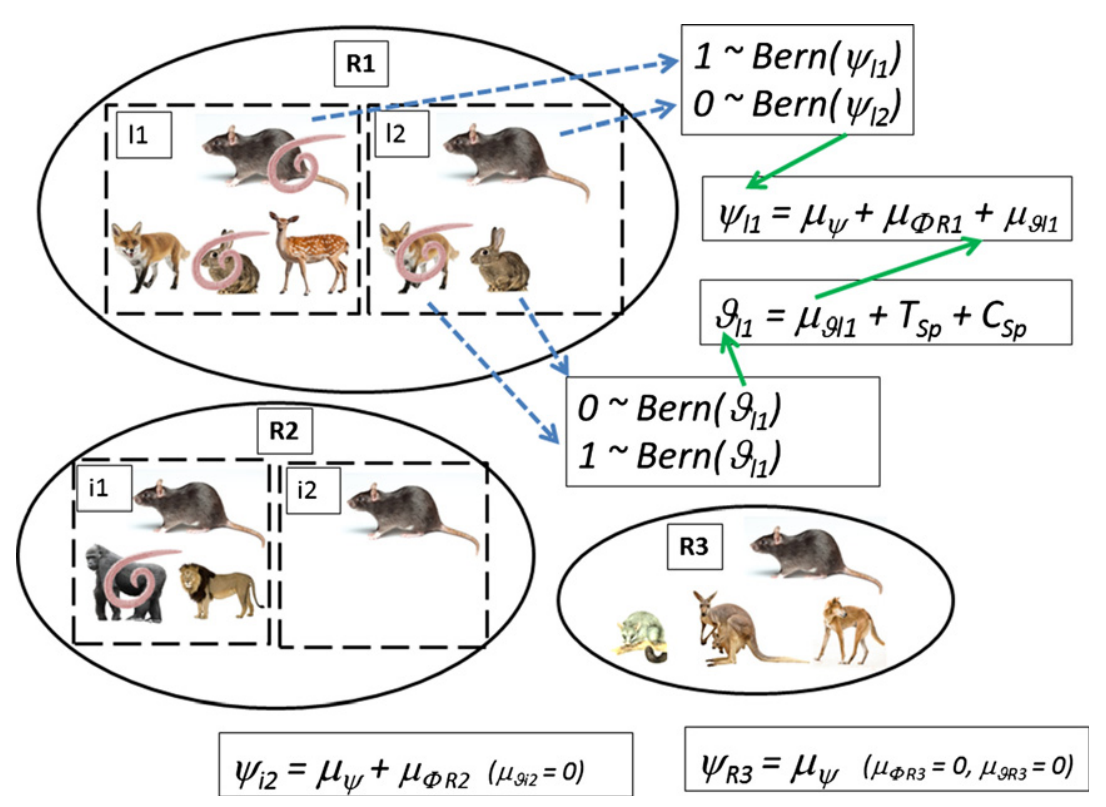

Figure 1 Illustration of the inverse Bayesian model for inferences on parasite geography and spillover effects from global species lists. The illustration represents a focal host species (dark rat) in three different regions ( $R 1-R 3$; illustrated wildlife species are examples from the Palaearctic, Afrotropical and Australian zoogeographic regions), which can be divided into any number of different locations ( $R 1: l 1$ and $l 2 ; R 2: i 1$ and $i 2$ ). Rats and also other mammals species have been sampled for a parasite species, which has been only found in a few species and localities, with presence recorded as ' 1 ' (nematode drawn on top of mammals) and absence as ' 0 '. Records are considered random draws from a Bernoulli distribution (blue arrows) with probabilities $\psi$ for the focal host species and probability $\vartheta$ for all other host species. Estimates of $\vartheta$ for any local host assemblage are used for the estimation of $\psi$, linking infection probability of local wildlife hosts to the focal host species (green arrows). The parasite has not been sampled from the focal host species in location $i 2$ and $R 3$. However, given the overall model framework, there is a certain probability that the focal species is also infected by the parasite in these areas: the intercept $\mu_{\psi}$ denotes an average global infection risk independent of region and location, while the parameter $\mu_{\Phi}$ estimates regional infection probability independent of location. Thus, $\mu_{\Phi} R 2>0$ (parasite is recorded in $i 1$ in $R 2$ ) and $\mu_{\Phi} R 3=0$ (no parasite recorded in $R 3$ ), and there is a higher probability that the parasite is present in $i 2$ than in $R 3$ given the data and parameter estimates. 
We assigned all locations to one of the 11 zoogeographic regions recently defined by Holt et al. (2013). We further assigned locations to the main climate zones (equatorial, arid, warm-temperate, snow, polar) based on an updated world map of the Köppen-Geiger climate classification (Kottek et al., 2006); if locations were covered by various climate zones (28 of 144), we assigned the relative proportion of the area covered by each climate zone and considered the uncertainty in which climate zones parasites were recorded with multiple data imputation as part of the Bayesian analysis and sampling procedure.

With the same approach, for each helminth species in our database we compiled the full range of host species for all locations from the NHML host-parasite database. For all mammal species in our database, we calculated the taxonomic distance to the genus 'Rattus' based on the number of nodes in a taxonomic tree (Wilson \& Reeder, 2005) resulting from the species' genus, family and order classification, indexed between 1 and 5. We further classified the IUCN conservation status of all mammal species (categories: least concern, near threatened, vulnerable, endangered, critically endangered) based on the 2001 assessment (version 3.1, http://www. iucnredlist.org). Note that we termed regional assemblages of mammals as 'wildlife hosts' in this study, but these assemblages also included humans and domestic mammals.

For data cleaning, all records not identified to species level were excluded, except those genera for which only single unidentified species were recorded. Scientific names were revised and standardized with the aid of a literature search in Thompson Reuters Web of Science (http://apps.webofknowledge.com/; latest searches performed in September 2013), personal literature collections and the mammal online database at http://vertebrates.si.edu/msw/mswCFApp/msw/ index.cfm (Wilson \& Reeder, 2005).

Our final data set for analysis included a total of 12,405 records of host-parasite association from different locations. Missing data were handled in our model approach by multiple data imputation. We are aware that our database is incomplete and lacks recently discovered helminth species. However, we do not consider this to be a problem, as we were interested in inference on geographic structure in hostparasite interactions from a finite data set, rather than complete lists of records. Species lists and classification of sampling locations are provided in Appendix S1 in the Supporting Information.

\section{Inferring host-parasite associations with an inverse modelling approach}

We used an inverse hierarchical modelling approach in a Bayesian framework to ask how likely it was for any parasite species to occur in a focal host species (Rattus rattus and $R$. norvegicus) in different locations inferred from a finite set of observations. To make inferential summary statistics on modelled estimates rather than observations, we estimated the probability of having a parasite species associated with a host species in any sampled location.

For all locations $l$, at which at least one parasite species $p$ has been recorded in at least one focal host species $h$, we assumed that all records $y(h, p, l)$ of host-parasite associations were random draws based on the true but unknown distribution of host-parasite associations such that

$$
y(h, p, l) \sim \operatorname{Bernoulli}(\psi(h, p, l))
$$

The probability of local host-parasite association $\psi(h, p, l)$ can be modelled further. In particular, we assumed $\psi(h, p, l)$ to be linked to the odds of the average occurrence probability of the respective parasite species $\Phi(p, r)$ within the zoogeographic region $r$ where $l$ is located (based on records from all kind of host species, irrespective of host species identity), given that locations from the same region are likely to harbour similar parasite assemblages. Likewise, we assumed $\psi(h, p, l)$ to be linked to the odds of the average occurrence probability of the respective parasite species $\Omega(p$, $c$ ) within the climate zone $c$ where $l$ is located. We also assumed $\psi(h, p, l)$ to vary with the average infestation probability of any mammal species from local assemblages with the same parasite, given as $\mu_{\vartheta}(p, l)$ (the odds of the infestation probability $\vartheta(p, l))$. Using a logit-link function, this gives:

$$
\begin{aligned}
\operatorname{logit} \psi(h, p, l)= & \mu_{\psi}(h, p)+\alpha_{1}(h, p) \mu_{\Phi}(p, r[l]) \\
& +\alpha_{2}(h, p) \mu_{\Omega}(p, c[l])+\alpha_{3}(h, p) \mu_{\vartheta}(p, l)
\end{aligned}
$$

where $\mu_{\psi}(h, p)$ is the species-specific intercept and $\alpha_{1}$ to $\alpha_{3}$ are coefficient estimates.

The covariates $\mu_{\Phi}(p, r), \mu_{\Omega}(p, c)$ and $\mu_{9}(p, l)$ are themselves considered as random variables (i.e. modelled probabilities from finite sets of observations), for which we assumed all observations, Фobs and $७$ obs respectively, as random draws out of the true but unknown parasite distributions and host associations. We thus assumed

$$
\begin{aligned}
& \operatorname{\Phi obs}(p, l) \sim \operatorname{Bernoulli}(\Phi(p, l)) \text { and } \\
& \vartheta \operatorname{obs}\left(p, l, x_{l}\right) \sim \operatorname{Bernoulli}(\vartheta(p, l))
\end{aligned}
$$

where $x_{l}$ indexes all mammal species examined in location $l$ for parasites.

We assumed again logit-link functions to model $\Phi(p, l)$ and $\vartheta(p, l)$ based on random intercepts such as

$$
\begin{aligned}
& \operatorname{logit} \Phi(p, l)=\mu_{\Phi}(p, r[l])+\mu_{\Omega}(p, c[l]) \text { and } \\
& \operatorname{logit} \vartheta(p, l)=\mu_{\vartheta}(p, l)+\gamma_{1} T(m)+\gamma_{2} C(m) .
\end{aligned}
$$

Here, we modelled $\vartheta(p, l)$ further as a function of speciesspecific taxonomic distance $T$ and their IUCN conservation status $C$ of mammal species $m ; \gamma_{1}$ and $\gamma_{2}$ are the respective coefficient estimates.

Given the estimated probability of local host-parasite association $\psi(h, p, l)$, we can express our uncertainty in the derived state variable $z(h, p, l)$ of whether a host species $h$ is 
infected with parasite species $p$ in location $l$ or not. This state is known for all positive recorded host-parasite associations (i.e. $z(h, p, l)=1$ ), whereas for all zero-records, we do not know this state as these may be true or 'false' zeros due to unobserved records of host-parasite associations. We thus assumed the unknown states $z(h, p, l)$ to be a random Bernoulli draw from estimated probabilities of host-parasite associations in different localities such that

$$
z(h, p, l) \sim \operatorname{Bernoulli}(\psi(h, p, l)) .
$$

The model was fitted in a Bayesian framework with Markov Chain Monte Carlo (MCMC) sampling based on the Gibbs sampler in the openbugs 3.2.2 software (Lunn et al., 2009). We used vague priors with uniform $U(0,100)$ for all variance terms and Gaussian distributed $N(0,1)$ for the model intercepts and coefficient estimates. The model code can be found in Appendix S2 in the Supporting Information. We assessed convergence and mixing of two parallel MCMC chains visually and obtained 5000 posterior MCMC samples after discarding 50,000 samples. For results, we calculated posterior mode and 95\% highest posterior density credible intervals (CI) from MCMC samples; CI for coefficient estimates not intersecting zero are interpreted as 'significant'.

\section{Calculating species richness and spatial turnover of parasite assemblages}

Repeated computation of the derived state variable $z(h, p, l)$ during MCMC sampling allowed us to infer on the most likely scenarios of species richness and spatial turnover of parasites in different host species and locations, with the variation in posterior estimates representing uncertainty in our inference. We were mostly interested in host-parasite associations at the scale of zoogeographic regions, and the presence/absence classification of host-parasite association at the scale of zoogeographic regions $z_{R}(h, p, r)$ is simply 1 if $z(h$, $p, l)=1$ for any location $l$ within $r$.

Species richness per host species at the scale of zoogeographic regions can then be calculated as the sum of all parasite species expected to be present such as

$$
S(h, r)=\sum_{p=1}^{P} z_{R}(h, p, r)
$$

For comparing parasite assemblages across zoogeographic regions and host species, we used $\beta_{\text {sim }}$ as a basic index of spatial turnover (Lennon et al., 2001), which is calculated as:

$$
\beta_{\text {sim }}=1-a /[\min (b, c)+a]
$$

based on the number of shared species $a$ between two samples and their numbers of unique species $b$ and $c$, respectively. We used this index as it focuses on composition with little bias from species richness. We compared the spatial turnover of parasite assemblages across zoogeographic regions from the same host $\beta_{\text {sim }}(h, r-r)$ and also among host species within different regions $\beta_{\text {sim }}(r, h-h)$.
For calculating the distinctiveness of host-parasite association mean $-\beta_{\text {sim }}(h, r)$ within host species in different zoogeographic regions, we calculated the mean of all pairwise $\beta_{\text {sim }}(h, r-r)$ estimates for the focal host species $h$ and zoogeographic region $r$ and all other regions with $r \in R$. We also tested for possible correlation between mean $-\beta_{\text {sim }}(h, r)$ with the distinctiveness of overall mammal assemblages based on the data of (Holt et al., 2013).

\section{RESULTS}

Our compiled database included a total of 241 helminth parasite species, of which 136 were recorded in Rattus rattus (species complex) and 164 in $R$. norvegicus; of these, 65 (27\%) parasite species were recorded in both $R$. rattus and R. norvegicus.

Parasite species associated with $R$. rattus and $R$. norvegicus were also recorded in as many as 718 other mammal species, of which at least 26 were endangered or critically endangered according to their IUCN conservation status in 2001. Further, $77(32 \%)$ of the helminth species were also recorded in humans.

Estimated species richness of parasitic helminths per zoogeographic region ranged from 1 (CI: 0-3, Madagascar) to 71 (CI: 67-76, Oriental) for $R$. rattus and 1 (CI: 0-3, Madagascar) to 100 (CI: 95-107, Palaearctic) for $R$. norvegicus. Estimating species richness and spatial turnover of host-parasite associations for sampling locations and regions revealed that records are rather incomplete, that is many host-parasite associations not recorded are likely to take place (Table 1).

Notably, differences in species richness estimates across different regions were correlated among the two host species (Pearson's correlation: $r=0.68$; Fig. 2). Likewise, estimates of spatial turnover $\beta_{\text {sim }}(h, r-r)$ of parasite assemblages across zoogeographic regions were significantly correlated for the two rat species (Mantel test across matrix of all combinations of different regions, Pearson's correlation $r=0.67$ ). For both rat species, $\beta_{\text {sim }}(h, r-r)$ varied between 0.14 (CI: 0.05-0.32) and 1.0 (CI: 0.4-1.0), suggesting clear biogeographic structure in parasite assemblages. The most distinct parasite assemblages for both rat species were estimated to be in the zoogeographic region of Madagascar with mean- $\beta_{\text {sim }}(h$, $r)=0.99(0.2-1)$ for both species (Fig. 3, Table 1). However, despite the correlation in species richness and spatial turnover among rat species, the similarity of parasite assemblages in the two rats from the same zoogeographic region was only moderate to negligible with $\beta_{\text {sim }}(r, h-h)$ estimates ranging between 0.24 (CI: 0.15-0.35) and 1 (CI: 0.49-1).

The estimated average infection probability of mammal species in the various sampling locations $\mu_{9}(p, l)$ had a significant positive impact on the infection probability for 114 of 241 parasite species in $R$. rattus and for 128 parasite species in $R$. norvegicus (i.e. lower limits of CI $>0$ for $\alpha_{3}$ ), indicating a clear link between host-parasite associations in the rats and the local wildlife assemblages. Variation in the 
Table 1 Summary of species richness and spatial turnover $\left(\right.$ mean $\left.-\beta_{\text {sim }}\right)$ of helminth parasite assemblages in the two host species Rattus rattus and $R$. norvegicus in different zoogeographic regions as defined by (Holt et al., 2013). For species richness, recorded numbers are given as $S_{\mathrm{Rec}}$, while posterior estimates are given as $S_{\text {Est. }}$. Spatial turnover estimates of mean $-\beta_{\text {sim }}$ are calculated as the mean of all pairwise $\beta_{\text {sim }}$ values from different locations within regions. $95 \%$ credible intervals for posterior estimates are given in parenthesis

\begin{tabular}{lrcl}
\hline Region & $S_{\text {Rec }}$ & \multicolumn{1}{l}{$S_{\text {Est }}$} & Mean- $\beta_{\text {sim }}$ \\
\hline R. rattus & & & \\
Afrotropical & 27 & $40(35-47)$ & $0.47(0.34-0.53)$ \\
Australian & 11 & $17(14-21)$ & $0.49(0.38-0.56)$ \\
Madagascan & 0 & $1(0-3)$ & $0.99(0.2-1)$ \\
Nearctic & 3 & $15(9-21)$ & $0.55(0.4-0.67)$ \\
Neotropical & 16 & $24(19-30)$ & $0.51(0.38-0.58)$ \\
Oceanian & 9 & $15(11-19)$ & $0.58(0.46-0.68)$ \\
Oriental & 64 & $71(67-76)$ & $0.38(0.24-0.43)$ \\
Palaearctic & 48 & $67(59-74)$ & $0.39(0.25-0.45)$ \\
Panamanian & 6 & $15(9-22)$ & $0.61(0.47-0.69)$ \\
Saharo-Arabian & 25 & $30(27-36)$ & $0.53(0.4-0.59)$ \\
Sino-Japanese & 15 & $28(22-34)$ & $0.56(0.42-0.63)$ \\
R. norvegicus & & & \\
Afrotropical & 0 & $24(17-33)$ & $0.58(0.45-0.68)$ \\
Australian & 13 & $19(16-23)$ & $0.49(0.36-0.55)$ \\
Madagascan & 0 & $1(0-3)$ & $0.99(0.3-1)$ \\
Nearctic & 27 & $34(30-43)$ & $0.54(0.41-0.59)$ \\
Neotropical & 19 & $29(24-35)$ & $0.59(0.44-0.65)$ \\
Oceanian & 0 & $11(5-16)$ & $0.54(0.38-0.69)$ \\
Oriental & 21 & $41(33-48)$ & $0.54(0.4-0.62)$ \\
Palaearctic & 97 & $100(95-107)$ & $0.26(0.21-0.4)$ \\
Panamanian & 6 & $14(9-20)$ & $0.58(0.44-0.67)$ \\
Saharo-Arabian & 26 & $33(28-38)$ & $0.53(0.4-0.58)$ \\
Sino-Japanese & 30 & $43(36-49)$ & $0.55(0.41-0.61)$ \\
\hline & & &
\end{tabular}

occurrence probability of parasites across climate zones $\mu_{\Omega}(p$, $c$ ) had a positive impact on the infection probability for only 7 of 241 parasite species in $R$. rattus and for eight parasite species in $R$. norvegicus (lower limits of CI $>0$ for $\alpha_{2}$ ).

Average infection probability of other mammal species with helminths decreased considerably with taxonomic distance from the genus Rattus (Fig. 4), and it also decreased with increasingly endangered status (according to their IUCN status) (Fig. 4). However, the species turnover in overall mammal assemblages in different zoogeographic regions was not correlated with the species turnover of parasite assemblages in the two rat species (both Mantel tests with Pearson's correlation coefficients $r<0.27)$.

\section{DISCUSSION}

Inferring host-parasite associations for two of the most common and invasive commensal rat species at a global scale showed that species richness and assemblage composition of parasitic helminths varied over zoogeographic regions. Geographic variation in parasite species richness and assemblage

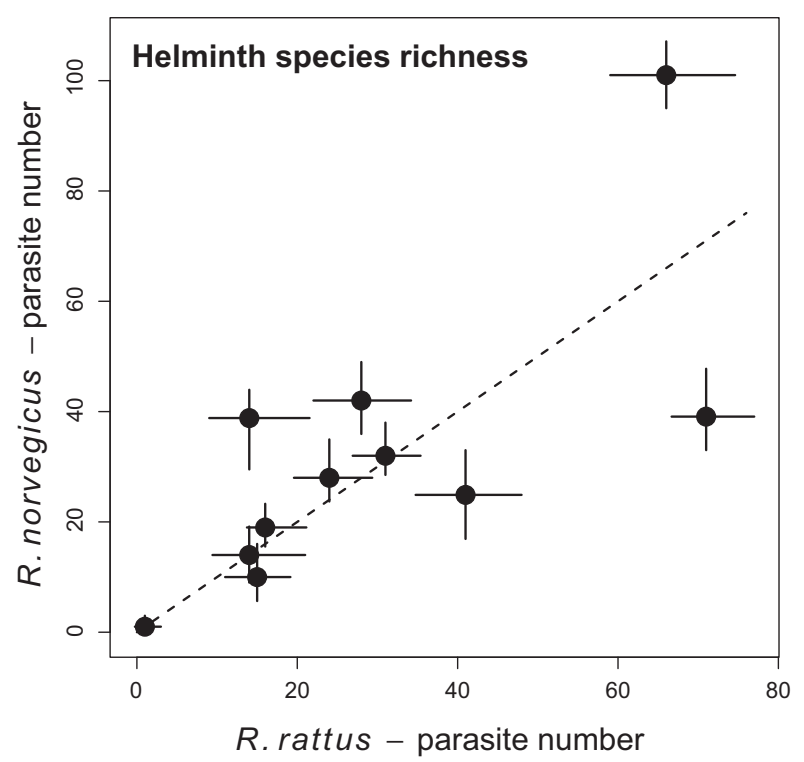

Figure 2 Relationship in the estimated numbers of helminth species associated with the two host species Rattus rattus and $R$. norvegicus in different zoogeographic regions given as posterior estimates of modes (points) and 95\% credible intervals (bars). The dashed line indicates a $1: 1$ relationship.

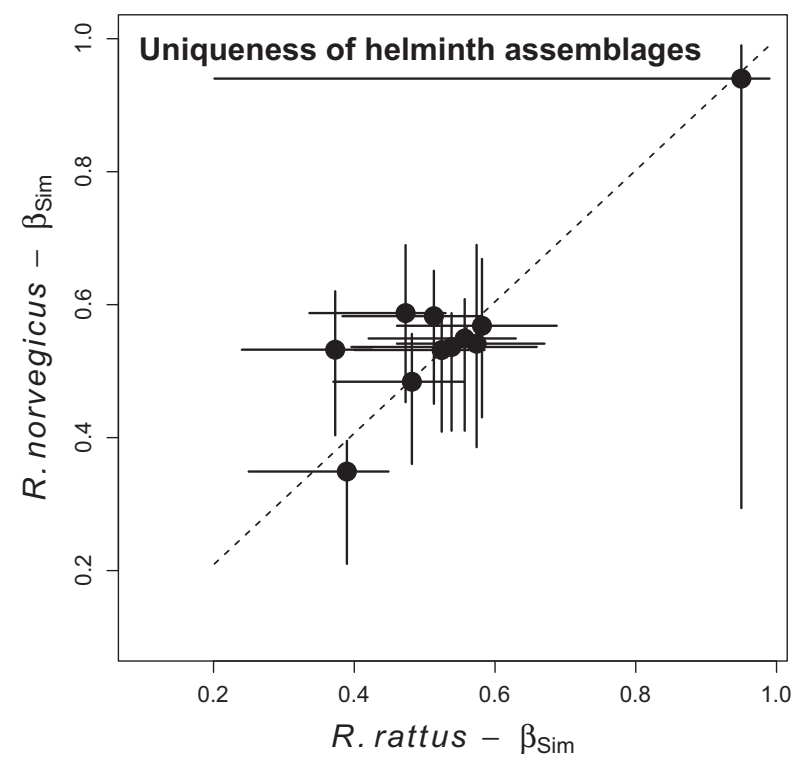

Figure 3 Distinctness of parasitic helminth assemblages associated with the two host species Rattus rattus and $R$. norvegicus in different zoogeographic regions as calculated from averaged spatial turnover estimates (modes of posterior samples are plotted as points and $95 \%$ credible intervals as bars). The dashed line indicates a $1: 1$ relationship.

composition was correlated between the two focal host species (Rattus rattus and $R$. norvegicus), although locally they were associated with distinct parasite assemblages. Further, our hierarchical model framework showed a clear influence of local species pools of wildlife hosts on parasite 

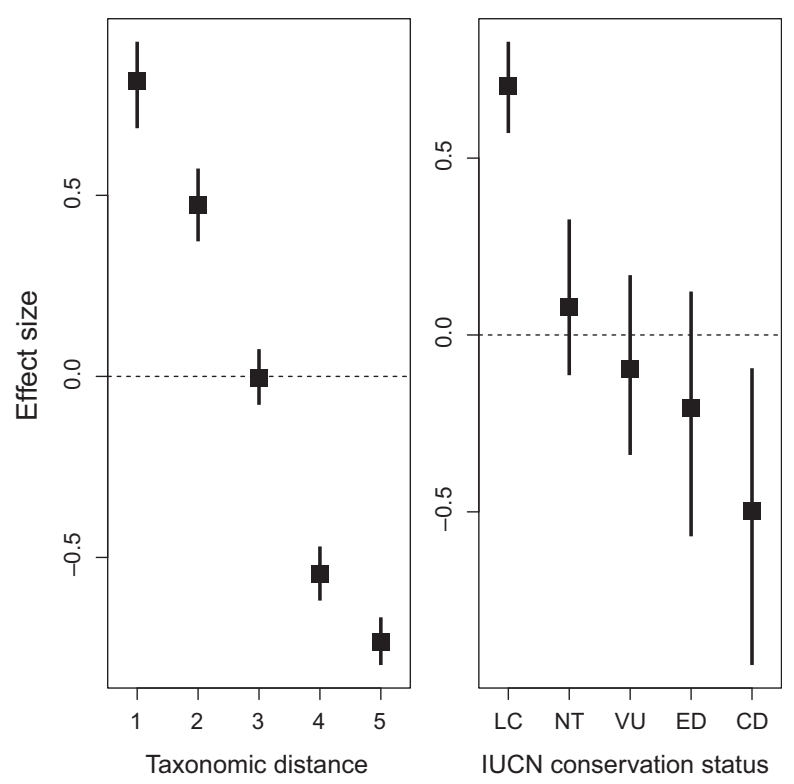

Figure 4 Posterior estimates of the relative impact of taxonomic distance from the genus 'Rattus' and the IUCN conservation status on the infestation probability of mammal species with the parasitic helminth species recorded in the two focal rat species Rattus rattus and $R$. norvegicus. Posterior modes are plotted as squares; $95 \%$ credible intervals as bars. Taxonomic distance indexed between 1 and 5 is based on species' genus, family and order classification; IUCN conservation status ranges from least concern (LC) to critically endangered (CD).

associations in the two focal host species, which supports the importance of spillover effects (Daszak et al., 2000). Moreover, in non-focal host species, taxonomic distance to the genus 'Rattus' and conservation status was related to the probability of being infected with a parasite species that had also infected one of the focal hosts.

Commensal rats have escaped several helminth parasites in regions such as Madagascar or Australia, where estimates of the species richness of parasites associated with the focal hosts are very small (see also Torchin et al., 2003). Only in the Palaearctic region were estimates of parasite species richness higher ( $R$. norvegicus) than in the Oriental region, where the host genus Rattus originated and diversified (Robins et al., 2008; Aplin et al., 2011). At a global scale, total numbers of recorded parasite species were considerably higher than those in the Oriental region for both focal host species, emphasizing that a considerable proportion of parasite species are linked to non-focal host species and were likely to have been acquired by the focal rat species during their invasion and colonization history. However, despite the clear link between focal and non-focal host-parasite associations, we do not know specifically which parasite species co-evolved with the rat species or any other host species. Moreover, with only general relationships in species richness and turnover examined, the underlying mechanisms that cause loss and acquisition of host-parasite association across geographic gradients remain unexplored.
Besides the likely impact of geographically varying regional wildlife host assemblages on parasites, there are likely to be other factors impacting parasite transmission and survival according to parasites' life histories. Parasitic helminths with either free-living stages in their life cycles or indirect transmission (e.g. via vectors) may be particularly sensitive to climate changes and other ecological perturbations (Brooks \& Hoberg, 2007), and variable conditions may result in geographic mosaics of species associations in time and space (Thompson \& Cunningham, 2002). Geographic patterns in host-parasite associations and other species interactions are most likely structured by multiple drivers of species and environmental attributes (Sheppard et al., 2010; Guilhaumon et al., 2012). Correlations in species richness and spatial turnover of parasites in the two focal host species, despite different associated assemblages, is an important result. However, additional studies are required to explore possible drivers of such relationships.

Contrary to our expectations, $R$. rattus was not associated with more parasite species than $R$. norvegicus nor did its associated parasite assemblages show more zoogeographic variation. Along with the findings that more closely related mammalian host species were more likely to be associated with the same parasite species, we conclude that parasite assemblages do evidently change with different conditions in zoogeographic regions but not necessarily with different habitat use of the focal host species, nor with their affinity for near-natural habitats shared with local wildlife host species. We found mammal species of least conservation concern were more likely to be infected with the parasites of the two rat species than endangered species. Most endangered species can be found in natural habitats that are at continuous decline due to human impact (Rondinini et al., 2011), whereas a large proportion of mammal species of least concern, including domestic species, are well able to persist in anthropogenic landscapes, where the focal hosts also occur. The stronger links between wildlife species of least conservation concern and the parasites recorded from the two commensal rats provide a first indication that habitat overlap and species ecological traits may impact the sharing of parasites between invasive species and local wildlife. However, we currently lack further detailed information to incorporate them into our analysis. Likewise, it is desirable to incorporate more geographic attributes of sample locations in future analysis to better partition the role of geography and ecology on the sharing of parasites by different host species (Davies \& Pedersen, 2008; Cooper et al., 2012).

Spillover and acquisition of parasites and pathogen are important in many ecological systems of wildlife and domestic or commercial species (Colla et al., 2006; Wood et al., 2012). Understanding the underlying mechanism for better predicting how particular species are under threat is typically challenged by disentangling geographical and ecological aspects. Pathogen transmission and spillover among species may include complex dynamics of the 'geographic' component: variation in the attraction of interacting species can 
induce spatio-temporal dynamics in spillover effects which are tightly linked to both phenological and ecological traits. Floral traits, for example, can determine pollinator attraction and the transmission of pathogens among pollinators such that infestation patterns of a single pollinator can only be understood when considering such multispecies interaction across geographic gradients (McArt et al., 2014). Inevitably, if model frameworks should match such multifaceted inference problem, they need to incorporate species and community-level aspects as well as the spatio-temporal context of interactions into a consistent framework. We expect hierarchical and inverse modelling approaches to be helpful for disentangling the various drivers in spillover dynamics.

Our study of parasite diversity in the two rat species is preliminary, as estimates of species richness and turnover are based on a finite sample size of collated data in which many parasite species are likely being missed. In particular, newly recognized parasite species were not included in our database. Some areas such as Madagascar are apparently poorly sampled, and we emphasize that precise estimates of true parasite species richness are hardly possible. Moreover, it remains unclear why certain parasite species are found in some areas but not in others. Nevertheless, previous work suggests that our Bayesian model approaches are capable of accurately accounting for uncertainty and unequal sample sizes when modelling species associations (Golicher et al., 2006; Wells \& O'Hara, 2013). Consequently, we expect the general inference about gradients in species richness and spatial turnover to be fairly robust. In contrast, it should be noted that estimates of the absolute number of parasite species are not exhaustive. In principle, our approach resembles other hierarchical models in ecology such as occupancy models (MacKenzie et al., 2002; Lachish et al., 2012) and multispecies regression approaches for modelling species interactions independent of sampling bias (Wells et al., 2013), in that we consider all zero-records as unknown states (i.e. they could be either a true zero or a missed observation). This approach could be equally well applied to investigations of disease emergence; as only if we distinguish possible natural patterns and processes from sampling effort can we distinguishing parasite and disease emergence from new records of previously overlooked parasites and diseases.

\section{CONCLUSIONS}

The geographic structure in parasite assemblages of commensal rats found in our study emphasizes that the emergence of such patterns can only be understood in relation to complex interactions linking commensal species and local wildlife hosts, from local to global scales. Such interactions include those with humans and domestic animals, which share many parasites and habitats with commensal rats.

As such multispecies host-parasite interactions take place along environmental and geographic gradients, analytical frameworks need to integrate species biological traits, the geography of parasites, the strength of spillover effects and environmental covariates to establish possible drivers of parasite loss and gain across organizational levels. In this way it is possible to link single host-species associations to community-wide patterns of associations, from local to global scale. Hierarchical model frameworks may provide a baseline for investigating the mechanisms underlying the spatio-temporal dynamics in such complex species interactions and distributions.

\section{ACKNOWLEDGEMENTS}

We are grateful to the Natural History Museum, London, in particular David Gordon, for online access to the host-parasite database. We thank Ben Holt for providing indices of zoogeographic species turnover in mammal assemblages and for extensive comments to an earlier draft. We thank Phil Lester, Dan Tompkins and an anonymous referee for contributing to this study with constructive comments.

\section{REFERENCES}

Aplin, K.P., Suzuki, H., Chinen, A.A. et al. (2011) Multiple geographic origins of commensalism and complex dispersal history of black rats. PLoS ONE, 6, e26357.

Boakes, E.H., McGowan, P.J.K., Fuller, R.A., Chang-Qing, D., Clark, N.E., O'Connor, K. \& Mace, G.M. (2010) Distorted views of biodiversity: spatial and temporal bias in species occurrence data. PLoS Biology, 8, e1000385.

Brooks, D.R. \& Hoberg, E.P. (2007) How will global climate change affect parasite-host assemblages? Trends in Parasitology, 23, 571-574.

Colla, S.R., Otterstatter, M.C., Gegear, R.J. \& Thomson, J.D. (2006) Plight of the bumble bee: pathogen spillover from commercial to wild populations. Biological Conservation, 129, 461-467.

Cooper, N., Griffin, R., Franz, M., Omotayo, M. \& Nunn, C.L. (2012) Phylogenetic host specificity and understanding parasite sharing in primates. Ecology Letters, 15, 1370-1377.

Daszak, P., Cunningham, A.A. \& Hyatt, A.D. (2000) Emerging infectious diseases of wildlife -threats to biodiversity and human health. Science, 287, 443-449.

Davies, T.J. \& Pedersen, A.B. (2008) Phylogeny and geography predict pathogen community similarity in wild primates and humans. Proceedings of the Royal Society B, 275, 1695-1701.

Estrada-Peña, A., Ostfeld, R.S., Peterson, A.T., Poulin, R. \& de la Fuente, J. (2014) Effects of environmental change on zoonotic disease risk: an ecological primer. Trends in Parasitology, 30, 205-214.

Gibson, D.I., Bray, R.A. \& Harris, E.A. (2005) Host-Parasite Database of the Natural History Museum, London. Natural History Museum, London. http://www.nhm.ac.uk

Golicher, D.J., O’Hara, R.B., Ruíz-Montoya, L. \& Cayuela, L. (2006) Lifting a veil on diversity: a Bayesian approach to fitting relative-abundance models. Ecological Applications, 16, 202-212. 
Goodman, S.M. (1995) Rattus on Madagascar and the dilemma of protecting the endemic rodent fauna. Conservation Biology, 9, 450-453.

Guilhaumon, F., Krasnov, B.R., Poulin, R., Shenbrot, G.I. \& Mouillot, D. (2012) Latitudinal mismatches between the components of mammal-flea interaction networks. Global Ecology and Biogeography, 21, 725-731.

Harris, D.B., Gregory, S.D. \& MacDonald, D.W. (2006) Space invaders? A search for patterns underlying the coexistence of alien black rats and Galápagos rice rats. Oecologia, 149, 276-288.

Hoberg, E.P. (2010) Invasive processes, mosaics and the structure of helminth parasite faunas. Revue Scientifique et Technique, 29, 255-272.

Holt, B.G., Lessard, J.-P., Borregaard, M.K., Fritz, S.A., Araújo, M.B., Dimitrov, D., Fabre, P.-H., Graham, C.H., Graves, G.R., Jønsson, K.A., Nogués-Bravo, D., Wang, Z., Whittaker, R.J., Fjeldså, J. \& Rahbek, C. (2013) An update of Wallace's zoogeographic regions of the world. Science, 339, 74-78.

Hopkins, M.E. \& Nunn, C.L. (2007) A global gap analysis of infectious agents in wild primates. Diversity and Distributions, 13, 561-572.

Hortal, J., Lobo, J.M. \& Jiménez-Valverde, A. (2007) Limitations of biodiversity databases: case study on seed-plant diversity in Tenerife, Canary Islands. Conservation Biology, 21, 853-863.

Jenkins, C.N., Pimm, S.L. \& Joppa, L.N. (2013) Global patterns of terrestrial vertebrate diversity and conservation. Proceedings of the National Academy of Sciences of the United States of America, 110, E2602-E2610.

Keating, K.A. \& Cherry, S. (2004) Use and interpretation of logistic regression in habitat selection studies. Journal of Wildlife Management, 68, 774-789.

Kottek, M., Grieser, J., Beck, C., Rudolf, B. \& Rubel, F. (2006) World map of the Köppen-Geiger climate classification updated. Meteorologische Zeitschrift, 15, 259-263.

Krasnov, B.R., Shenbrot, G.I., Khokhlova, I.S. \& Degen, A.A. (2004) Relationship between host diversity and parasite diversity: flea assemblages on small mammals. Journal of Biogeography, 31, 1857-1866.

Lachish, S., Gopalaswamy, A.M., Knowles, S.C.L. \& Sheldon, B.C. (2012) Site-occupancy modelling as a novel framework for assessing test sensitivity and estimating wildlife disease prevalence from imperfect diagnostic tests. Methods in Ecology and Evolution, 3, 339-348.

Lennon, J.J., Koleff, P., Greenwood, J.J.D. \& Gaston, K.J. (2001) The geographical structure of British bird distributions: diversity, spatial turnover and scale. Journal of Animal Ecology, 70, 966-979.

Lomolino, M.V. (2004) Conservation biogeography. Frontiers of Biogeography: New Directions in the Geography of Nature (ed. by M.V. Lomolino and L.R. Heaney), pp. 293-296. Sinauer Associates, Sunderland, MA.

Lunn, D., Spiegelhalter, D., Thomas, A. \& Best, N. (2009) The BUGS project: evolution, critique and future directions. Statistics in Medicine, 28, 3049-3067.
MacKenzie, D.I., Nichols, J.D., Lachman, G.B., Droege, S., Royle, J.A. \& Langtimm, C.A. (2002) Estimating site occupancy rates when detection probabilities are less than one. Ecology, 83, 2248-2255.

Martin, T.G., Wintle, B.A., Rhodes, J.R., Kuhnert, P.M., Field, S.A., Low-Choy, S.J., Tyre, A.J. \& Possingham, H.P. (2005) Zero tolerance ecology: improving ecological inference by modelling the source of zero observations. Ecology Letters, 8, 1235-1246.

McArt, S.H., Koch, H., Irwin, R.E. \& Adler, L.S. (2014) Arranging the bouquet of disease: floral traits and the transmission of plant and animal pathogens. Ecology Letters, 17, 624-636.

Meerburg, B.G., Singleton, G.R. \& Kijlstra, A. (2009) Rodent-borne diseases and their risks for public health. Critical Reviews in Microbiology, 35, 221-270.

Morand, S. (2012) Phylogeography helps with investigating the building of human parasite communities. Parasitology, 139, 1966-1974.

Morand, S. \& Krasnov, B.R. (2010) The Biogeography of Host-Parasite Interactions. Oxford University Press, Oxford.

Morand, S., McIntyre, K.M. \& Baylis, M. (2014) Domesticated animals and human infectious diseases of zoonotic origins: domestication time matters. Infection, Genetics and Evolution, 24, 76-81.

Ostfeld, R.S. \& Keesing, F. (2012) Effects of host diversity on infectious disease. Annual Review of Ecology, Evolution, and Systematics, 43, 157-182.

Patz, J.A., Graczyk, T.K., Geller, N. \& Vittor, A.Y. (2000) Effects of environmental change on emerging parasitic diseases. International Journal for Parasitology, 30, 1395-1405.

Poulin, R. \& Mouillot, D. (2003) Host introductions and the geography of parasite taxonomic diversity. Journal of Biogeography, 30, 837-845.

Reese, G.C., Wilson, K.R., Hoeting, J.A. \& Flather, C.H. (2005) Factors affecting species distribution predictions: a simulation modeling experiment. Ecological Applications, 15, 554-564.

Robins, J.H., McLenachan, P.A., Phillips, M.J., Craig, L., Ross, H.A. \& Matisoo-Smith, E. (2008) Dating of divergences within the Rattus genus phylogeny using whole mitochondrial genomes. Molecular Phylogenetics and Evolution, 49, 460-466.

Rondinini, C., di Marco, M., Chiozza, F., Santulli, G., Baisero, D., Visconti, P., Hoffmann, M., Schipper, J., Stuart, S.N., Tognelli, M.F., Amori, G., Falcucci, A., Maiorano, L. \& Boitani, L. (2011) Global habitat suitability models of terrestrial mammals. Philosophical Transactions of the Royal Society B: Biological Sciences, 366, 2633-2641.

Sheppard, S.K., Colles, F., Richardson, J., Cody, A.J., Elson, R., Lawson, A., Brick, G., Meldrum, R., Little, C.L., Owen, R.J., Maiden, M.C.J. \& McCarthy, N.D. (2010) Host association of Campylobacter genotypes transcends geographic variation. Applied and Environmental Microbiology, 76, 5269-5277. 
Singleton, G.R., Sudarmaji \& Brown, P.R. (2003) Comparison of different sizes of physical barriers for controlling the impact of the rice field rat, Rattus argentiventer, in rice crops in Indonesia. Crop Protection, 22, 7-13.

Stenseth, N., Leirs, H., Skonhoft, A., Davis, S., Pech, R., Andreassen, H., Singleton, G., Lima, M., Machang'u, R., Makundi, R., Zhang, Z., Brown, P., Shi, D. \& Wan, X. (2003) Mice, rats, and people: the bio-economics of agricultural rodent pests. Frontiers in Ecology and the Environment, 1, 367-375.

Taylor, L.H., Latham, S.M. \& Woolhouse, M.E.J. (2001) Risk factors for human disease emergence. Philosophical Transactions of the Royal Society B Biological Sciences, 356, 983989.

Thieltges, D.W., Hof, C., Dehling, D.M., Brändle, M., Brandl, R. \& Poulin, R. (2011) Host diversity and latitude drive trematode diversity patterns in the European freshwater fauna. Global Ecology and Biogeography, 20, 675-682.

Thompson, J.N. \& Cunningham, B.M. (2002) Geographic structure and dynamics of coevolutionary selection. Nature, 417, 735-738.

Torchin, M.E., Lafferty, K.D., Dobson, A.P., McKenzie, V.J. \& Kuris, A.M. (2003) Introduced species and their missing parasites. Nature, 421, 628-630.

Ward, G., Hastie, T., Barry, S., Elith, J. \& Leathwick, J.R. (2009) Presence-only data and the em algorithm. Biometrics, 65, 554-563.

Wells, K. \& O'Hara, R.B. (2013) Species interactions: estimating per-individual interaction strength and covariates before simplifying data into per-species ecological networks. Methods in Ecology and Evolution, 4, 1-8.

Wells, K., O’Hara, R.B., Pfeiffer, M., Lakim, M.B., Petney, T.N. \& Durden, L.A. (2013) Inferring host specificity and network formation through agent-based models: tickmammal interactions in Borneo. Oecologia, 172, 307-316.

Wells, K., Lakim, M.B. \& O’Hara, R.B. (2014) Shifts from native to invasive small mammals across gradients from tropical forest to urban habitat in Borneo. Biodiversity and Conservation, 23, 2289-2303.
Wilson, D.E. \& Reeder, D.M. (2005) Mammal Species of the World. A Taxonomic and Geographic Reference, 3rd edn. Johns Hopkins University Press, Baltimore.

Wood, J.L.N., Leach, M., Waldman, L., MacGregor, H., Fooks, A.R., Jones, K.E., Restif, O., Dechmann, D., Hayman, D.T.S., Baker, K.S., Peel, A.J., Kamins, A.O., Fahr, J., Ntiamoa-Baidu, Y., Suu-Ire, R., Breiman, R.F., Epstein, J.H., Field, H.E. \& Cunningham, A.A. (2012) A framework for the study of zoonotic disease emergence and its drivers: spillover of bat pathogens as a case study. Philosophical Transactions of the Royal Society B, 367, 2881-2892.

\section{SUPPORTING INFORMATION}

Additional Supporting Information may be found in the online version of this article:

Appendix S1 List of parasite species and sampled locations.

Table S1 List of parasitic helminth species and associated number of mammal hosts.

Table S2 Assignments of sampled locations to zoogeographic regions.

Appendix S2 Model code in BUGS language.

\section{BIOSKETCHES}

The authors of this study have various interests linked to biodiversity, parasitology, conservation, species distributions, ecohealth, biotic interactions, and hierarchical models, and assembled as a multidisciplinary team. All authors contributed jointly to this study, mostly by asking naïve questions to each other that helped to critically scrutinize approaches and synthesize different views into this study.

Editor: Jacqueline Beggs 